\title{
Gas-Liquid Phenomena with Dynamic Contact Angles in the Cathode of a Parallel Proton Exchange Membrane Fuel Cell
}

\author{
Xichen Wang, Biao Zhou ${ }^{1}$, Mengcheng Jiang \\ Department of Mechanical, Automotive and Materials Engineering \\ University of Windsor \\ Windsor, ON, Canada, N9B 3P4
}

\begin{abstract}
Among the numerical studies on liquid water transport in Proton Exchange Membrane Fuel Cells (PEMFCs), the static contact angle (SCA) model is generally used, while the dynamic contact angle (DCA) model is only applied on the simulation of droplet behaviors with simple geometries like a single microchannel. In this study, the DCA model is employed to study the gas-liquid phenomena inside the cathode of PEMFC with parallel flow field design, i.e., Parallel-DCA model. The water emerging and transport processes are simulated based on this Parallel-DCA model using the volume of fluid (VOF) method. The numerical results are also compared to those from the Parallel-SCA model with the same computational domain and operating conditions.
\end{abstract}

Keywords-proton exchange membrane fuel cell; volume of fluid; dynamic contact angle; parallel.

\section{INTRODUCTION}

As the main product in the cathode, water plays an important role in the performance of a proton exchange membrane fuel cell (PEMFC). Too much or too little liquid water will result in an increase of voltage loss, which is considered to be a key point affecting the performance of PEMFCs. Therefore, water management, particularly liquid water management, is a significant technical issue in the optimization of the PEMFC performance, which has received further attention in this research area.

Comparing with existing numerical models employed in the simulation of gas-liquid dynamics in PEMFCs, the volume of fluid (VOF) model, which was proposed by Quan et al. [1], has the advantage of tracking the interface between the liquid and the gas so that the liquid water distribution and transport can be described in detail. Recently, for the numerical studies on twophase flow in PEMFCs, the VOF method has been widely used and recognized as an effective tool in this area and a series of research works have been reported [2-9].

However, all the aforementioned numerical studies only considered static contact angle (SCA) as wall boundary condition. It is known that the geometric dimensions of PEMFCs are quite small, namely from a microscopic view, wall adhesion and surface tension significantly affect the

${ }^{1}$ Corresponding Author and Principal Investigator: Dr. Biao Zhou, bzhou@uwindsor.ca, 1-519-253-3000 ext. 2630 gas/liquid distribution and transport, thus the contact angle is unlikely to stay at equilibrium. Djilali et al. $[10,11]$ noted that liquid water behavior on the GDL surface is affected by the contact angle when water emerges from a pore. Consequently, in the numerical models of PEMFCs (unsteady, two-phase flow model), it is not reliable to use static contact angle (SCA) as the wall boundary condition. Sikalo et al. [12] conducted experimental and numerical studies for droplet impact on horizontal surface, and it is revealed that there are obvious limitations using SCA as one of the boundary conditions and the DCA should be considered. The Hoffman function [13], an empirical correlation for DCA (also known as Kistler's Law), was employed in [12] to predict the contact angle values:

$$
\begin{gathered}
\theta_{d}=f_{\text {Hoff }}\left[C a+f_{\text {Hoff }}^{-1}\left(\theta_{s}\right)\right] \\
f_{\text {Hoff }}(x)=\arccos \left\{1-2 \tanh \left[5.16\left(\frac{x}{1+1.31 x^{0.99}}\right)^{0.706}\right]\right\}
\end{gathered}
$$

In (1), $C a$ is the capillary number and $f_{\mathrm{Hoff}}^{-1}\left(\theta_{S}\right)$ is the shift factor obtained from the inverse of the Hoffman function under SCA [14]. Hoffman function is considered as one of the promising formulae in DCA simulations [15]. However, so far, nearly all the numerical models with DCAs are for simple geometries and use the DCA only for a single wall boundary [16-18]. Recently, Jiang et al. [15] found that the AR-DCA model, which is implemented with Hoffman function and considers both advancing and receding dynamic contact angles, can well simulate droplet behaviors and dynamic wetting behaviors. This AR-DCA model is validated against a series of experiments for droplet impact on horizontal and inclined surfaces from Sikalo et al. [19, 20], showing its potential to be applied in the simulation of gas-liquid phenomena in PEMFCs.

In this study, we extend our previous work [4] by applying DCAs at multiple wall boundaries to investigate the gas-liquid phenomena inside the cathode of a parallel PEMFC, i.e., Parallel-DCA model. The general liquid water transport processes are presented and discussed, and also compared to those from Parallel-SCA model. 


\section{NUMERICAL MODEL DESCRIPTION}

\section{A. Numerical Simulation Domain}

The numerical simulation domain for the parallel model represents the geometry of a full-scale cathode side of a single PEMFC, as shown in Fig. 1. The detailed dimensions and descriptions of this domain can be found in [4].

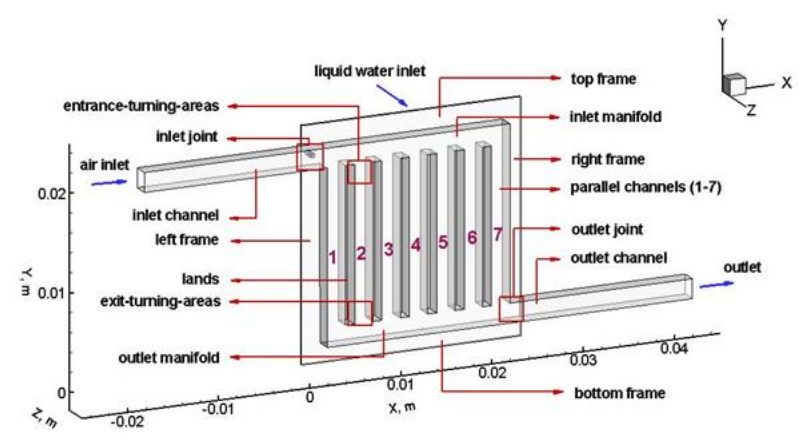

Figure 1. Computational domain of Parallel model.

\section{B. Computational Methodology}

A three-dimensional PEMFC cathode model is developed in this study to investigate gas-liquid phenomena and water transport processes. Air is modeled in the gaseous phase and liquid water is modeled in the liquid phase; these two-phases are assumed to be immiscible. The VOF method is applied to track the liquid-gas flow interface. The governing equations and corresponding computational methodology were reported in our previous work by Wang and Zhou [4]. The method to implement DCA model was reported by Jiang and Zhou [15]. More details and descriptions can be found in [4] and [15].

\section{Boundary Conditions and Grid Independency}

The accelerated model [3] is employed in this study by adjusting the mass flow rate of air and liquid water, which can significantly save the computation time. The mass flow rate for the liquid water inlet and gas inlet is set as $1.7 \times 10^{-4} \mathrm{kgs}^{-1}$ and $2.0 \times 10^{-5} \mathrm{kgs}^{-1}$ respectively. The initial contact angle for the side walls of channels, upper walls of channels and walls of porous layer are $40^{\circ}, 43^{\circ}$ and $140^{\circ}$ respectively. In ParallelSCA model, the contact angles are constant for the wall boundaries, whereas in the Parallel-DCA model, the contact angles vary with the change of the flow field and can be expressed as parameters associated with time and location.

The Parallel-DCA model and Parallel-SCA model [4] share the same computational domain and the mesh set up and independency has also been reported in [4].

\section{RESULTS AND DISCUSSION}

\section{A. General Process of Liquid Water Transport in Parallel- DCA Model}

The general process of liquid water transport in the ParallelDCA model, as shown in Fig. 2, can be summarized into several sub-processes:

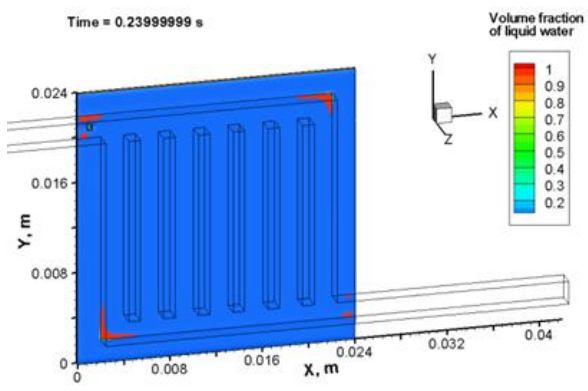

(a)

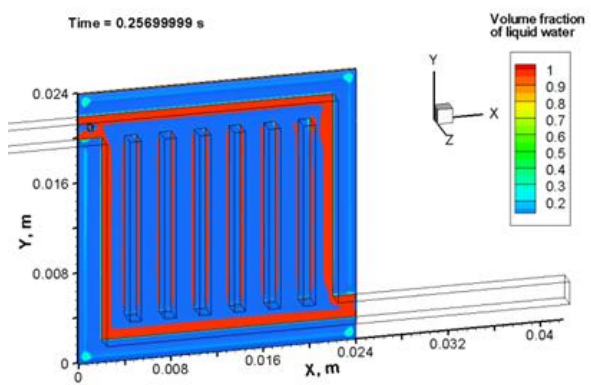

(b)

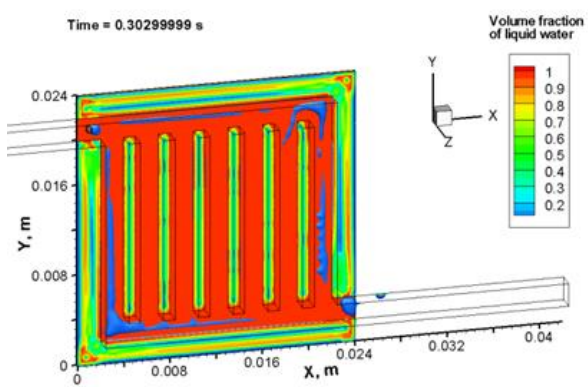

(c)

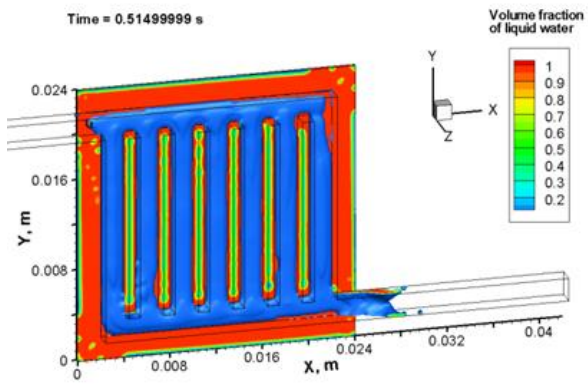

(d)

Figure 2. General liquid water transport in the Parallel-DCA model.

1) Liquid water is supplied from the back surface of the porous layer with a constant mass flow rate to simulate the liquid water generation through the electrochemical reaction.

2) The liquid water reaches the top surface of the porous layer, first accumulates at the four corners of the interface under the channels domain (Fig. 2(a)), and then the frame and the edges of each parallel channel (Fig. 2(b)).

3) Liquid water from the porous layer emerges into the gas flow channels first from the interface under the frame of the channels domain, in the middle area of the channels (Fig. 3(c)). 
4) More liquid water emerges into the channels. With time, channels become flooded and liquid water starts to drain out through the outlet channel, as shown in Fig. 2(d).

In summary, the general process of water transport and removal in the Parallel-DCA model are similar to those of the Parallel-SCA model [4] in the early stage (Fig. 2(a) and (b)). However, some different phenomena can be observed: for the Parallel-DCA model, the liquid water emerges into the gas channels first from the middle area of the interface under the channels, whereas for the Parallel-SCA model, the liquid water emerges into the gas channels from the peripheral edges of the lands and the peripheral edges of the channels domain. The detailed comparisons are provided and illustrated in the following sections.

\section{B. Comparison of Emerging Process}

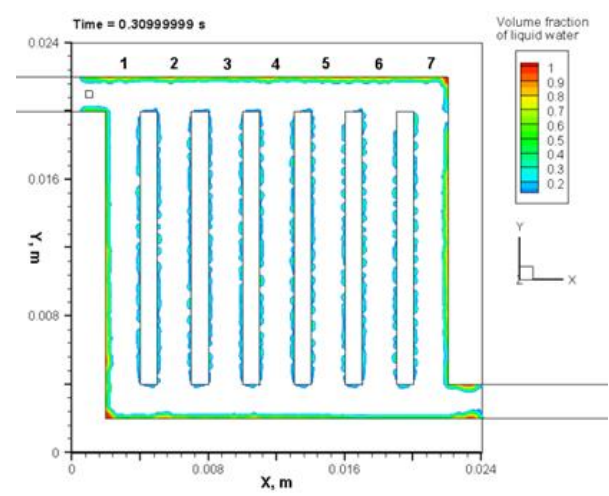

(a) Parallel-SCA model.

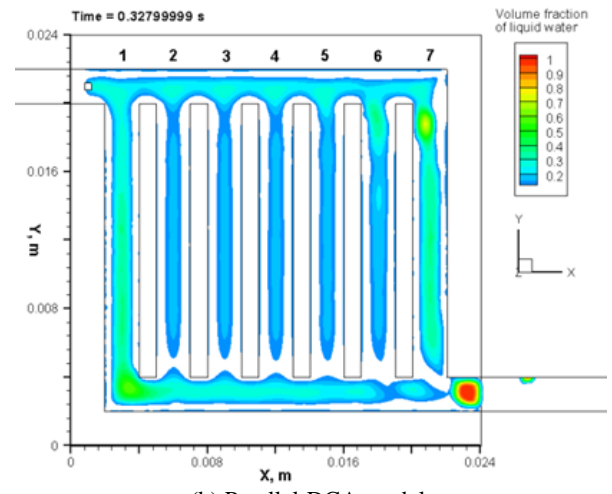

(b) Parallel-DCA model.

Figure 3. Comparison of the liquid water distribution near interface at $\mathrm{Z}=$ $0.00031 \mathrm{~m}$.

Fig. 3 (a) and (b) show the liquid water emerging pattern in the gas flow channels from Parallel-SCA and Parallel-DCA models respectively, located at the plane extracted near the interface $(Z=0.00031 \mathrm{~m})$ from the channel domain. It can be further observed that for Parallel-SCA model, the liquid water enters into the gas flow channels from the peripheral edges of the channel domain and emerges from the land edges (Fig. 3(a)); whereas for Parallel-DCA model, the liquid water mainly passes through the interface under the middle area, with only a small amount of water emerging from the edges of the lands and moving along the sidewalls (Fig. 3(b)).
The detailed emergence and growth of liquid water from the porous layer into the channels is presented by Fig. 4, which shows the liquid water volume fraction with different reference velocity vectors located at the No. 7 parallel channel on the plane (extracted from the computational domain at $\mathrm{Y}=0.012$ $\mathrm{m}$ as illustrated in Fig. 4(a)). For Parallel-SCA model, due to the uniform water generation rate on the back surface of the porous layer, the liquid water from the porous layer enters into the channels from the land edges; it can also be observed that there is a pair of vortices - an upper vortex and a lower one in the cross-section of the parallel channels, referred to as Dean vortices, which normally occur in curved channels or pipes [21]. For Parallel-DCA model, the liquid water emerges from the porous layer enters into the channel at the middle area, and the pair of vortices has a left-right distribution.

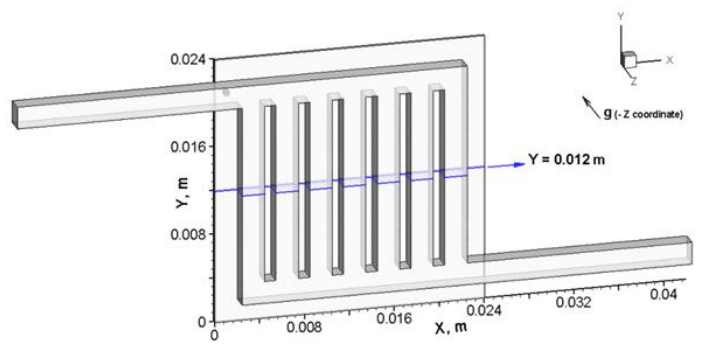

(a) Schematic of extracted plane.

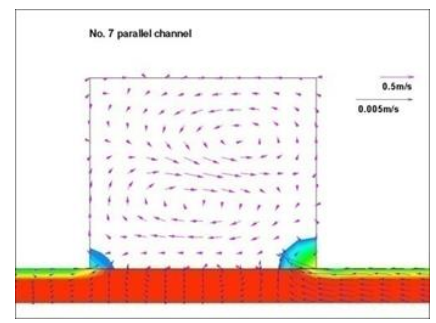

(b) Parallel-SCA model.

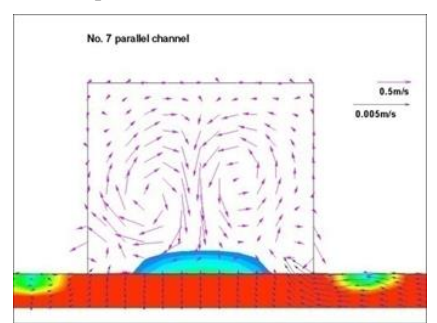

(c) Parallel-DCA model.
Figure 4. Comparison of liquid water emerging on plane at $\mathrm{Y}=0.012 \mathrm{~m}$ (enlarged plane with velocity vectors).

\section{Comparison of Flow Pattern in the Draining Process}

The draining process in the Parallel-SCA and ParallelDCA models can be explained by Fig. 5, which shows the deformation of the draining liquid water in the outlet channel. For the Parallel-SCA model, valley-shaped water near the outlet joint will deform into a top-bottom stratified flow pattern while moving along the outlet channel to drain out, as shown in Fig. 5(a); whereas for the Parallel-DCA model, the deformation of the liquid water in the outlet channel is in a stratified-slug pattern, as shown in Fig. 5(b). Also, it can be observed that there are several splashed, small droplets in the outlet channel adhered to the sidewalls of the outlet channel, because their inertial force is not strong enough to overcome the friction or wall adhesion. 


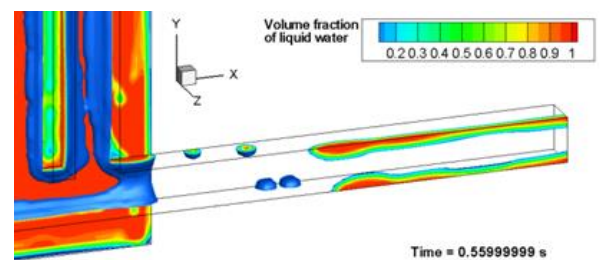

(a) Parallel-SCA model.

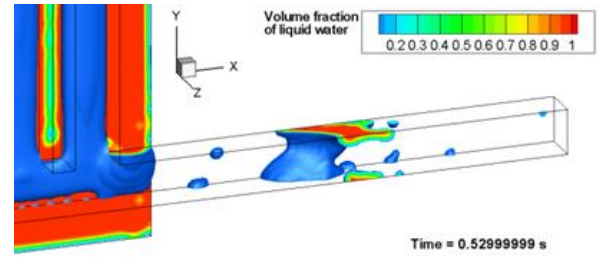

(b) Parallel-DCA model.

Figure 5. Comparison of the flow pattern in the draining process.

\section{CONCLUSIONS}

In this study, the Parallel-DCA model is proposed by applying DCA at multiple wall boundaries in a PEMFC cathode. The gas-liquid phenomena and water transport processes are simulated and investigated using the ParallelDCA model and VOF method. The numerical results are compared to those from the Parallel-SCA model [4]. It is indicated that although the general water transport processes from these two models are similar, there are several differences observed from the phenomena that can be summarized as follows:

(1) Emerging pattern (SCA model: liquid water first emerges from the edges of the interface under the channels; DCA model: liquid water first emerges from the middle of the interface under the channels).

(2) Dean vortices evolution (SCA model: upper-lower distribution; DCA model: left-right distribution).

(3) Draining deformation (SCA model: top-bottom stratified flow; DCA model: stratified-slug flow).

In the future, we will further investigate the potential of the DCA model to be applied in the simulation of gas-liquid phenomena in PEMFCs with other complex flow field designs.

\section{ACKNOWLEDGMENT}

The authors are grateful for the support from the Natural Sciences and Engineering Research Council of Canada (NSERC) and the University of Windsor.

\section{REFERENCES}

[1] Quan P, Zhou B, Sobiesiak A, Liu Z. Water behavior in serpentine micro-channel for proton exchange membrane fuel cell cathode. Journal of Power Sources. 2005 Dec 1;152:131-45.
[2] Le AD, Zhou B. A numerical investigation on multi-phase transport phenomena in a proton exchange membrane fuel cell stack. Journal of Power Sources. 2010 Aug 15;195(16):5278-91.

[3] Jiao K, Zhou B. Accelerated numerical test of liquid behavior across gas diffusion layer in proton exchange membrane fuel cell cathode. Journal of Fuel Cell Science and Technology. 2008 Nov 1;5(4):041011.

[4] Wang X, Zhou B. Liquid water flooding process in proton exchange membrane fuel cell cathode with straight parallel channels and porous layer. Journal of Power Sources. 2011 Feb 15;196(4):1776-94.

[5] Ding Y, Anderson R, Zhang L, Bi X, Wilkinson DP. Simulations of twophase flow distribution in communicating parallel channels for a PEM fuel cell. International Journal of Multiphase Flow. 2013 Jun 30;52:3545.

[6] Jo JH, Kim WT. Numerical simulation of water droplet dynamics in a right angle gas channel of a polymer electrolyte membrane fuel cell. International Journal of Hydrogen Energy. 2015 Jul 13;40(26):8368-83.

[7] Qin Y, Li X, Jiao K, Du Q, Yin Y. Effective removal and transport of water in a PEM fuel cell flow channel having a hydrophilic plate. Applied Energy. 2014 Jan 31;113:116-26.

[8] Kang S, Zhou B. Numerical study of bubble generation and transport in a serpentine channel with a T-junction. International Journal of Hydrogen Energy. 2014 Feb 4;39(5):2325-33.

[9] Kang S, Zhou B, Jiang M. Bubble behaviors in direct methanol fuel cell anode with parallel design. International Journal of Hydrogen Energy. 2017 Aug 3;42(31):20201-15.

[10] Zhu X, Sui PC, Djilali N. Numerical simulation of emergence of a water droplet from a pore into a microchannel gas stream. Microfluidics and Nanofluidics. 2008 Jun 1;4(6):543-55.

[11] Zhu X, Sui PC, Djilali N. Three-dimensional numerical simulations of water droplet dynamics in a PEMFC gas channel. Journal of Power Sources. 2008 Jun 15;181(1):101-15.

[12] Šikalo Š, Wilhelm HD, Roisman IV, Jakirlić S, Tropea C. Dynamic contact angle of spreading droplets: Experiments and simulations. Physics of Fluids. 2005 Jun;17(6):062103.

[13] Kistler SF. Hydrodynamics of wetting. Wettability. 1993;6:311-430.

[14] Hoffman RL. A study of the advancing interface. I. Interface shape in liquid_-gas systems. Journal of Colloid and Interface Science. 1975 Feb $1 ; 50(2): 228-41$.

[15] Jiang M, Zhou B, Wang X. Comparisons and validations of contact angle models. International Journal of Hydrogen Energy. 2018 Mar 2.

[16] Miller C. Liquid water dynamics in a model polymer electrolyte fuel cell flow channel, MASc Thesis, University of Victoria, 2009.

[17] Fang C, Hidrovo C, Wang FM, Eaton J, Goodson K. 3-D numerical simulation of contact angle hysteresis for microscale two phase flow. International Journal of Multiphase Flow. 2008 Jul 31;34(7):690-705.

[18] Jiang M, Zhou B. Numerical Study of Droplet Impact on Inclined Surface: Viscosity Effects. ECS Transactions. 2018 Jan 4;83(1):127-36.

[19] Šikalo Š, Tropea C, Ganić EN. Impact of droplets onto inclined surfaces. Journal of Colloid and Interface Science. 2005 Jun 15;286(2):661-9.

[20] Šikalo S̆, Ganić EN. Phenomena of droplet-surface interactions. Experimental Thermal and Fluid Science. 2006 Nov 30;31(2):97-110.

[21] Dean WR. XVI. Note on the motion of fluid in a curved pipe. The London, Edinburgh, and Dublin Philosophical Magazine and Journal of Science. 1927 Jul 1;4(20):208-23. 